(2) OPEN ACCESS

- Additional material is published online only. To view please visit the journal online (http://dx.doi.org/10.1136/ tobaccocontrol-2020-055658).

${ }^{1}$ Demography Department, Faculty of Spatial Sciences, University of Groningen, Groningen, The Netherlands ${ }^{2}$ Netherlands Interdisciplinary Demographic Institute - KNAW/ University of Groningen, The Hague, The Netherlands

\section{Correspondence to}

Prof Fanny Janssen, Demography Department, Faculty of Spatial Sciences, University of Groningen, Groningen, The Netherlands; F.Janssen@rug.nl

Received 23 January 2020 Revised 23 April 2020 Accepted 3 June 2020

\title{
Smoking epidemic in Europe in the 21st century
}

\author{
Fanny Janssen 지 , ${ }^{1,2}$ Shady El Gewily, ${ }^{1}$ Anastasios Bardoutsos ${ }^{1}$
}

\author{
ABSTRACT \\ Objective To estimate smoking-attributable mortality \\ in the long-term future in 29 European countries using \\ a novel data-driven forecasting approach that integrates \\ the wave pattern of the smoking epidemic and the \\ cohort dimension.
}

Methods We estimated and forecasted age-specific and age-standardised smoking-attributable mortality fractions (SAMF) and 95\% projection intervals for 29 European countries by sex, 1950-2100, using age-period-cohort modelling with a generalised logit link function. We projected the (decelerating) period increases (women) by a quadratic curve to obtain future declines, and extrapolated the past period decline (men). In addition, we extrapolated the recent cohort trend. Results SAMF among men are projected to decline from, on average, 25\% in 2014 (11\% (Sweden) - 41\% (Hungary)) to $11 \%$ in 2040 (range: 6.3\%-15.4\%), 7\% in 2065 (range: $5.9 \%-9.4 \%$ ) and $6 \%$ in 2100. SAMF among women in 21 non-Eastern European countries, currently at an average of $16 \%$, are projected to reach peak levels in 2013 (Northern Europe), 2019 (Western Europe), 2027 (Greece, Italy) and 2022 (Central Europe), with maximum levels of, on average, 17\% (8\% (Greece)-28\% (Denmark)), and to decline to $10 \%$ in 2040 (range: $4 \%-20 \%$ ), 5\% in 2065 (range: $3.5 \%-7.6 \%$ ) and $4 \%$ in 2100 . For women, a short-term shift in the peak of the inverse U-shaped age pattern to higher ages is projected, and crossovers between the age-specific trends.

Conclusion Our novel forecasting method enabled realistic estimates of the mortality imprint of the smoking epidemic in Europe up to 2100. The high peak values in smoking-attributable mortality projected for women warrant attention.

\section{INTRODUCTION}

Smoking is the leading preventable mortality risk factor in Europe. ${ }^{1}$ The smoking prevalence among adults in Europe is the highest worldwide, and the share of all-cause mortality due to smoking (16\% among adults aged 30 and over) in Europe is higher than the global share of $12 \% .^{2}$ For public health policy-makers, knowing how the smoking epidemic and its mortality imprint (smoking-attributable mortality) is likely to develop in the future is highly relevant.

Long-term estimates of future smokingattributable mortality for European countries are, however, rare, and a simple extrapolation from past trends is not sufficient. As the descriptive smoking epidemic model has demonstrated, past trends in smoking-attributable mortality follow a waveshaped pattern of increase (eventually) followed by a decelerating decline, reflecting trends in the increase and decline in smoking prevalence some
30-40 years earlier. ${ }^{34}$ For European men, smokingattributable mortality is already declining, whereas for women, smoking-attributable mortality is still increasing, except in selected north-Western European countries. ${ }^{5}$ Furthermore, important differences between birth cohorts in smoking-attributable mortality exist reflecting the long-term effects of generational differences in the uptake of smoking. ${ }^{67}$

Previous comprehensive cause-of-death projections provided valuable information on future total smoking-attributable deaths in the world and its regions over the short term, but not for individual European countries. ${ }^{8} 9$ To our knowledge, more recent cause-of-death projections ${ }^{10-13}$ have not been used to generate future smoking-attributable mortality estimates.

Two previous projections of smoking-attributable mortality in the long-term future for European countries used an age-period-cohort approach, in line with the more common lung cancer mortality forecasts, ${ }^{14-17}$ which they semi-quantitatively extended to include insights from the smoking epidemic model. ${ }^{18} 19$ However, their estimates focused on individual forerunner countries, and their methodology cannot easily be extended to other countries. ${ }^{19} \mathrm{Li}$ and Raftery very recently forecasted smoking-attributable mortality up to 2050 in 69 countries using a quantitative data-driven approach that consists of a joint model for men and women and generates a wave-shaped pattern. ${ }^{20}$ However, they ignored the cohort dimension, and projected smoking-attributable mortality only for all ages combined.

We provide estimates of smoking-attributable mortality in the long-term future, both age-specific and for all ages combined, by sex for 29 European countries using a novel, purely data-driven approach that takes into account both the wave pattern of the smoking epidemic and the cohort dimension.

\section{METHODOLOGY}

See online supplementary file 1 for more details.

\section{Data}

We studied national populations by sex and age (35-84 for men; 40-84 for women) for 29 European countries with long-term (lung cancer) mortality data, from 1950 onwards. See online supplementary appendix table 1 . Consequently, we studied the cohorts born between approximately 1866 (1950 minus 84) and 1980 (2015 minus 35).

We estimated for these sex-specific national populations, age $(x)$-specific and time $(t)$-specific smoking-attributable mortality fractions $\left(\mathrm{SAMF}_{x, t}\right)$ (=the share of all-cause mortality due to smoking). We did so by applying the commonly used indirect Peto-Lopez method, ${ }^{20} 21$ in simplified form. ${ }^{518}$ 
The Peto-Lopez method uses national lung cancer mortality rates, controlled for the part not due to smoking, as a proxy for past smoking intensities. SAMF ${ }_{x, t}$ are subsequently obtained by applying to these past smoking intensities the relative risks (=RRs) of dying from smoking, ${ }^{22}$ corrected for the exposure of smokers to other risk factors. Whereas the original PetoLopez method applies the RRs for different causes of death, ${ }^{21}$ the simplified method applies, at once, the RR for all causes combined. ${ }^{518}$

The national lung cancer mortality rates were calculated by dividing observed lung cancer deaths by country, year, sex and 5 -year age groups $(0-4, \ldots, 75-79,80+)$-obtained from $\mathrm{WHO}^{23}$ - with respective population exposures from the Human Mortality Database (HMD). ${ }^{24}$ Using Loess smoothing, we obtained the rates and the $\mathrm{SAMF}_{x, t}$ by single year of age.

We estimated time comparable smoking-attributable mortality fractions across adult ages $\left(\mathrm{SAMF}_{t}\right)$ by standardising for the population-specific age-distribution of deaths in 2010, obtained from the HMD. ${ }^{25}$

\section{Methodology}

To estimate future SAMF while accounting for the wave pattern of the smoking epidemic and the cohort dimension, we applied age-period-cohort (APC) modelling, and we used the fact that a wave pattern for the fractions is obtained when the logit of the fractions (=logistically transformed fractions) has a quadratic shape.

More specifically, we applied the APC modelling approach by Cairns $e t \mathrm{al}^{26}$ to the fractions while using a generalised logit link function. The generalisation enabled us to restrict the projected fractions (and their projection intervals (PIs)) to feasible future values (eg, not zero and in line with the theory) by implementing lower and upper bounds (LB, UB).

The model we applied is $\operatorname{logit}\left(\frac{\operatorname{SAMF}_{x, t}-\mathrm{LB}[x]}{\mathrm{UB}[x]-\mathrm{LB}[x]}\right)=\alpha_{x}+\kappa_{t}+\gamma_{t-x}$ The parameters $\alpha_{x}, \kappa_{t}$ and $\gamma_{t-x}$ capture the age pattern ax, the overall time trend kt and the cohort patterns gc, respectively.

For women, we ensured that the projected peak SAMF level would be lower than the peak SAMF level for men in the same country by implementing as UBs the maximum observed agespecific SAMF levels among Danish women over the years 1951-2014. ${ }^{527}$ For men, we implemented a theoretical UB representing the sex-specific and age-specific SAMF associated with $100 \%$ prevalence and a RR of dying from smoking of 2.25 .522

We implemented LBs in line with the presumed lower limits and asymmetric wave pattern in the smoking epidemic model. ${ }^{34}$ We assumed a future minimum smoking prevalence of $5 \%$ for both men and women, based on evidence that current smoking prevalence levels are fairly similar for men and women in the forerunner countries, ${ }^{28} 29$ a smoking prevalence of $8.7 \%$ in Sweden in $2014,{ }^{28}$ and the recent emphasis on smoking prevention policies. ${ }^{30-34}$ The associated age-standardised SAMF bounds are $5.9 \%$ for men and $3.5 \%$ for women.

To obtain the age-specific SAMF LB values, we applied to the age-standardised SAMF bounds the country-specific shape of the age pattern of SAMF among men in 2014, which we assume to reflect the age pattern close to the final stage of the smoking epidemic for both men and women.

For women, the implementation of the age-specific LBs proved challenging because their levels were higher than many past observed levels. For women in the majority of countries this challenge could be dealt with by a slight adjustment of the procedure. Unfortunately, no realistic long-term projections could be obtained for women in Portugal, Spain, Belarus, Estonia, Latvia, Lithuania, Russia and Ukraine.

We performed a sensitivity analysis where we did not implement the LBs.

For women, we projected the (decelerating) increases in the period parameter kt deterministically by a quadratic curve with correlated errors to obtain future declines. A quadratic curve in the logit of fractions will result in a wave pattern in the normal fractions. The start year of $\mathrm{kt}$ in the projections is the first year in which $\mathrm{kt}$ increases.

For men, we extrapolated the linear decline in kt after the peak by time series forecasting using AutoRegressive Integrated Moving Average (ARIMA) models. ${ }^{35} \mathrm{~A}$ linear decline in the logit of fractions will result in a deceleration of the decline in the normal fractions. We projected kt for men from maximally 10 years after its peak by applying the best-fitting ARIMA model $(\mathrm{p}, \mathrm{d}=1, \mathrm{q})$ with drift, based on minimum AICc (Akaike Information Criterion corrected for small sample sizes), with $\mathrm{p}$ and $\mathrm{q} \leq 3$.

We projected the cohort parameter gc for women in all countries and for men in 14 countries by extrapolating the recent decline of the observed inverse U-shape by applying the procedure that was used for projecting kt among men. For men in Austria, Netherlands, Denmark, Norway, Switzerland, United Kingdom (UK), Sweden, Finland and Luxembourg, for whom the inverse U-shape was followed by a recent increase or stagnation, we assumed a future stable level, implemented using an ARIMA $(0,1,0)$ model with no drift. For men in the Czech Republic, Poland, Russia, Ukraine, Belarus and Slovakia, we extrapolated the fluctuating gc trend using the best-fitting mean reverting ARIMA (p,0,q) model with $\mathrm{p} \leq 3$ and $\mathrm{q} \leq 2$. We projected the cohort parameter only after excluding outer birth cohorts for whom the estimates were not statistically significant (women) or the trends were very volatile (men).

We projected median age-specific and sex-specific SAMF up to 2100 , and estimated their $95 \%$ PIs by performing 50000 simulations. Point estimates (medians) and PIs for the age-standardised SAMF are obtained by age-standardising over each sample path separately.

We validated our method with back-tests for men in the Netherlands and men in Denmark, using data up to 1975 to project up to 2015 (online supplementary figure S6). Our method can accurately predict the year of the maximum age-standardised SAMF and its level, and the $\mathrm{SAMF}_{x, t}$ at low and middle ages. At higher ages, however, our projections resulted in lower values than those observed from 2000/2005 onwards, which resulted in an underestimation of future age-standardised SAMF. Li and Raftery observed a similar underestimation of $\mathrm{SAMF}_{t}$ in their validation for the Netherlands using data up to 2000 to project up to $2015 .^{20}$

\section{RESULTS}

Past trends in age-standardised SAMF (men: ages 35-84; women: ages 40-84) (figure 1) illustrate the clear wave pattern of increases followed by declines among men. Among women in most countries, except in selected north-Western European countries, SAMF is still increasing. Among men, the peak in SAMF was, on average, reached in 1989, at 35\% (table 1). In 2014, SAMF levels were, on average, $25 \%$ among men, ranging from $11 \%$ in Sweden to $41 \%$ in Hungary; and $12.5 \%$ among women, ranging from $0.1 \%$ in Belarus to $27 \%$ in Denmark (table 1).

In line with the smoking epidemic theory, our projections show a deceleration of the decline among men, and a reversal 

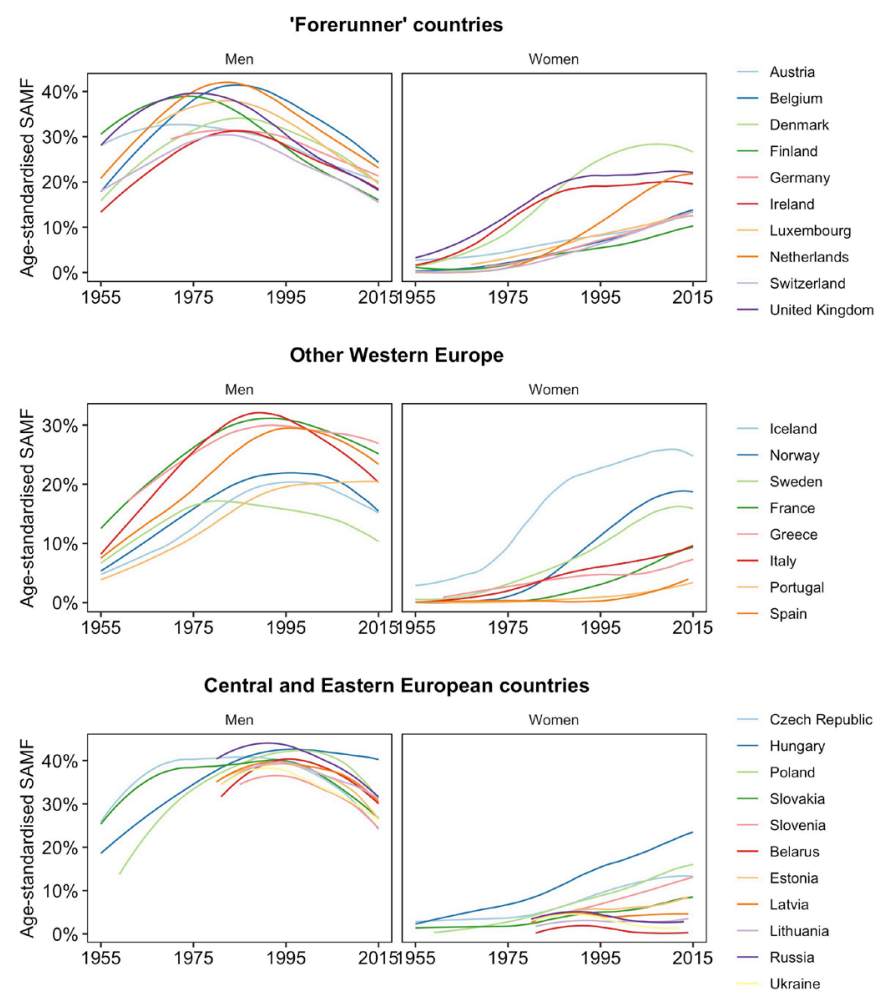

Figure 1 Past trends in smoothed* age-standardised smokingattributable mortality fractions (SAMF) (men: ages 35-84; women: ages 40-84), 29 European countries, from 1950 onwards, by sex and region. *Loess smoothing (degree $=2$; span $=0.25$ ) of observed with projected. For populations without projections, Loess smoothing (degree $=2$ and span $=0.75$ ) of observed.

from an increase to a decelerating decline among women (table 1, figures 2 and 3).

In 2040, SAMF levels for men are projected to be, on average, $11 \%$, ranging from $6.5 \%$ (Estonia; Sweden) to 15\% (Hungary; Portugal) (table 1). This represents a large reduction from the current level of $25 \%$. The average levels and range are expected to further decline to $7.2 \%$ (range: $5.9 \%-9.4 \%$ ) in 2065 and to $6.3 \%$ in 2100 , in line with the LB of $5.9 \%$.

For women, an important outcome is the projected peak year of SAMF and the associated level. For women in Northern Europe, the peak has already been reached in all countries except Finland, and on average, the peak is expected to occur in 2013 (ranging from 2006 in Denmark to 2029 in Finland), at a level of $20 \%$ (ranging from $28 \%$ in Denmark to $12 \%$ in Finland). For women in Western Europe, the peak has already been reached only in Ireland and the UK. On average, the peak is expected to occur in 2019 (ranging from 2009 in Ireland to 2032 in Austria), at a level of $16 \%$ (ranging from around $22 \%$ in the UK and the Netherlands to $10 \%$ in France). For women in Greece and Italy, the peak is expected to occur, on average, in 2027, at $11 \%$. For women in the five included Central European countries, the peak is projected to occur, on average, in 2022 (ranging from 2013 in the Czech Republic to 2034 in Slovenia), at 16\% (ranging from $9 \%$ in Slovakia to 26\% in Hungary). Across the 21 countries for which reliable projections could be made, the maximum level of SAMF is, on average, $17 \%$, ranging from $8 \%$ in Greece to $28 \%$ in Denmark.

For women, SAMF levels are projected to be, on average, $10 \%$ in 2040, ranging from 20\% (Hungary) to slightly under 5\%
(Sweden, France, Greece, Slovakia), 4.6\% (range: 3.5\%-7.6\%) in 2065 and, finally, $4.0 \%$ in 2100 .

For Austria, Belgium, Denmark, Iceland, Ireland, Italy, Norway, Slovenia, Sweden and the UK SAMF levels are projected to be substantially higher among women than men over the short term (figure 2).

For men a clear further convergence in SAMF levels can be observed, although that Sweden continues to experience considerably lower levels until 2035, and Hungary considerably higher levels up to 2040 (figure 3). Among women, also convergence is projected, especially when ignoring Hungary. However, the projected differences between countries in both 2030 and 2040 are larger compared with men, especially in relative terms. This is because the (projected) peak among women is expected to occur relatively late in some countries (eg, Slovenia, Italy, Austria, Finland) while in other-especially Northern European-countries SAMF is-by then-already declining for a number of years.

The projections of age-specific SAMF (online supplementary appendix figure 1) show, for men, declines at all ages, and a final convergence of age-specific SAMF levels. For women, by contrast, a crossover between the wave-shaped trends for the different age groups is projected, in line with the cohort dimension and previous age-period-cohort forecasts of lung cancer mortality. ${ }^{16}$

For men, the inverse U-shaped age pattern is not projected to change (online supplementary appendix figure $2 \mathrm{a}$ ). For women, the peak of the age pattern, currently around age 50-60, is first shifted to higher ages due to cohort differences in the uptake of smoking, ${ }^{18} 19$ and is then shifted to younger ages (online supplementary appendix figure $2 \mathrm{~b}$ ), in line with the assumed similar age pattern as currently observed for men.

\section{DISCUSSION}

\section{Extension of the smoking epidemic model}

Our projections facilitate the extension of the smoking epidemic model $^{34}$ for Europe up to the end of the 21st century (figure 4). For men in the 29 European countries studied, we project a continued deceleration of the decline in age-standardised SAMF (age 35-84) from the average peak of 35\% in 1989, and an average level of $25 \%$ in 2014 , to an average level of $11 \%$ in 2040, 7\% in 2065 and 6\% in 2100. SAMF levels (age 40-84) among women are projected to first increase and then decline. More specifically, among women in the 21 non-Eastern European countries for which we could obtain realistic projections, SAMF, which is currently, on average, 16\% (12.5\% for all 29 countries), is projected to reach its peak on average 30-35 years later than among men, at a level of $17 \%$ which is close to half of men's level. Subsequently, the average SAMF levels among women are expected to decline at a decelerating pace to $10 \%$ in 2040, 5\% in 2065 and 4\% in 2100; thereby staying below men's average SAMF levels.

There are, however, important differences between the European regions and between individual European countries, particularly among women, that should not be ignored. SAMF among women is projected to (have) reach(ed) peak levels, on average, in Northern Europe first $(2013 ; 20 \%)$, then in Western Europe $(2019 ; 16 \%)$ and later in the five Central European countries (2022; 16\%). For women in Slovenia (2034), Italy (2033), Austria (2032) and Finland (2029), our median projections indicate that the peak in the mortality impact will not be reached for more than a decade (table 1). In 2040, SAMF levels are projected to be $\geq 10 \%$ in the majority of countries for both 
Table 1 Current and future (maximum) age-standardised smoking-attributable mortality fractions (SAMF) (men: 35-84; women: 40-84), selected years $2014^{*}-2100,29$ European countries, by country or region and sex

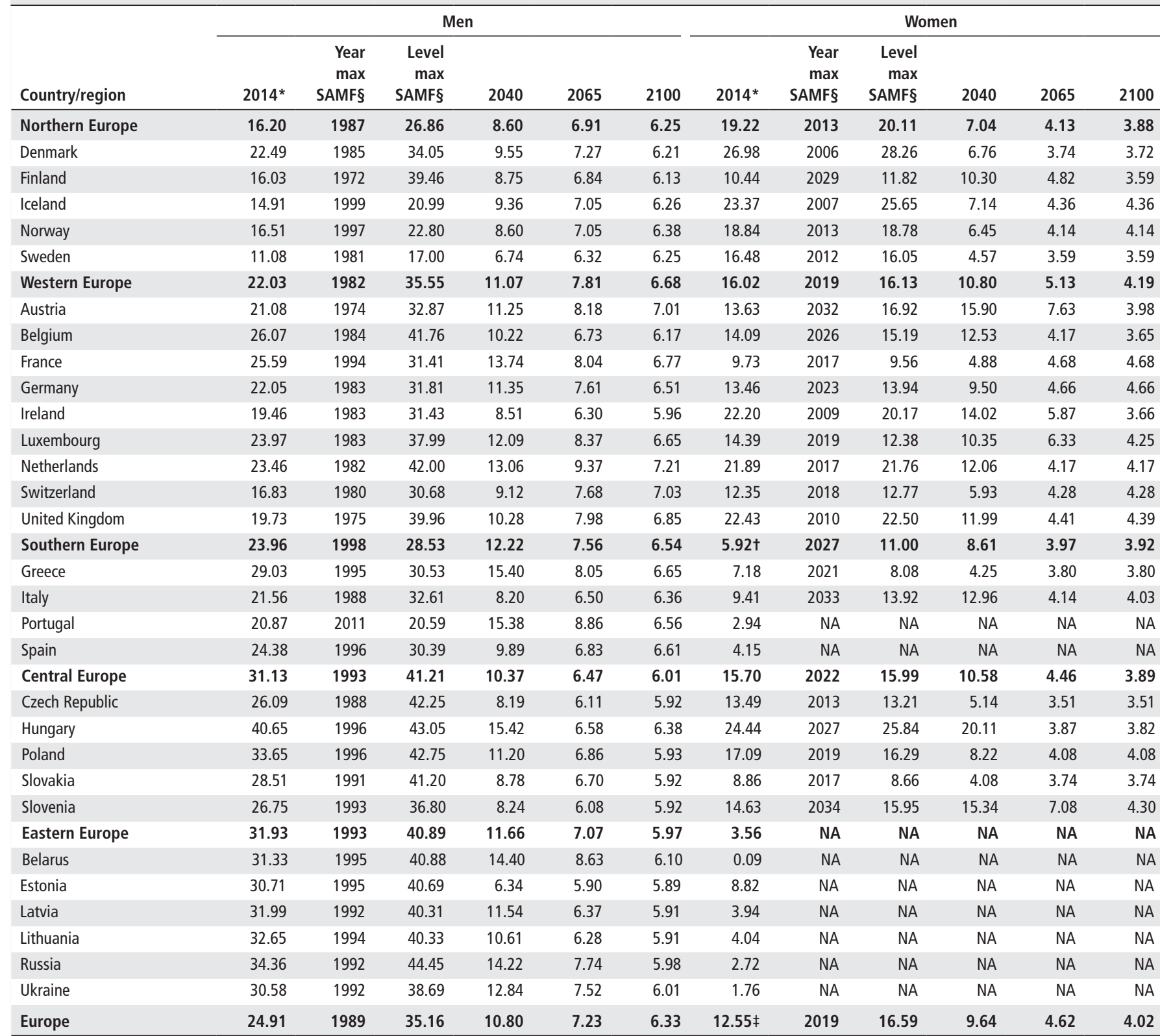

Bold indicates the unweighted averages for the regions.

*Or the latest available year before that for Greece (2013), Russia (2013) and Ukraine (2012).

†Average based on the four included Southern European countries. For Greece and Italy the average is $8.30 \%$.

¥Average based on the 29 included countries. For the 21 countries with realistic projections, the average is $15.97 \%$.

$\S$ Based on smoothing (Loess 2/0.25) over time of the fitted (or observed when absent) with the projected values.

men and women, and even $\geq 15 \%$ in Greece (men), Portugal (men), Austria (women) and Slovenia (women), but especially in Hungary. This whereas in other countries, and particularly in Sweden, very low levels of SAMF levels are already projected for 2040 (table 1).

Trends in (projected) SAMF reflect the trends in smoking prevalence about 30-40 years earlier. ${ }^{34}$ Similarly, the average onset of the decline in SAMF among women around 2019, is in line with declines in smoking prevalence among women in the majority of European countries from at least 1990 onwards. ${ }^{36}$ Among women in the UK, Denmark and the Netherlands, declines in smoking prevalence were occurring already in the 1970s. ${ }^{19}$ Thus, for selected north-Western European countries, the peak in SAMF has already occurred. Among men, declines in

smoking prevalence have been occurring over a more extended period. $^{319}$

Similarly, the country differences in projected SAMF levels in 2040 largely reflect recently observed differences in smoking prevalence. For example, in 2014 smoking prevalence was still as high as $25.8 \%$ in Hungary, but was as low as $8.7 \%$ in Sweden. ${ }^{28}$

\section{Reflection on the methodology}

In line with previous projections of smoking-attributable mortality, ${ }^{19} 20$ our projection uses indirect estimates of smokingattributable mortality based on the frequently applied indirect Peto-Lopez method. ${ }^{21}$ The use of an indirect estimation technique avoids relying on incomplete detailed historical smoking 

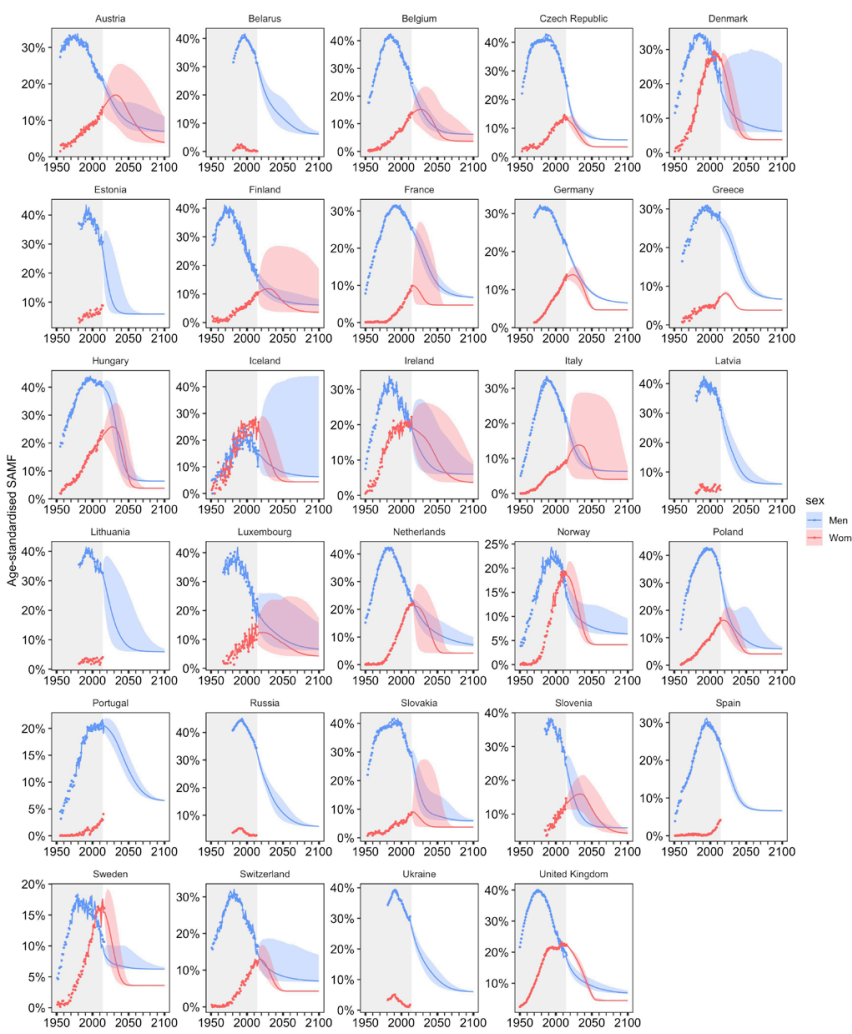

Figure 2 Past and future trends in age-standardised smokingattributable mortality fractions (SAMF) (men: 35-84; women: 40-84), 1950-2100, 29 European countries, by country and sex.

prevalence data, facilitates the use of high-quality cause-ofdeath information, and enables capturing the effects not only of smoking prevalence, but of smoking duration and smoking intensity. See Janssen for a detailed discussion of the simplified indirect Peto-Lopez method we employed. ${ }^{5}$ Although the SAMF levels would be affected by any estimation method, earlier research indicated that the estimation of future trends and the projected peak year of SAMF among women were less likely to be affected. ${ }^{19}$

We incorporated the important cohort dimension of smokingattributable mortality into our forecasting approach using age-period-cohort (APC) modelling. We used the Cairns et al approach ${ }^{26}$ which builds on the commonly applied Clayton and Schifflers approach, ${ }^{37}$ but includes an additional step (beneficial
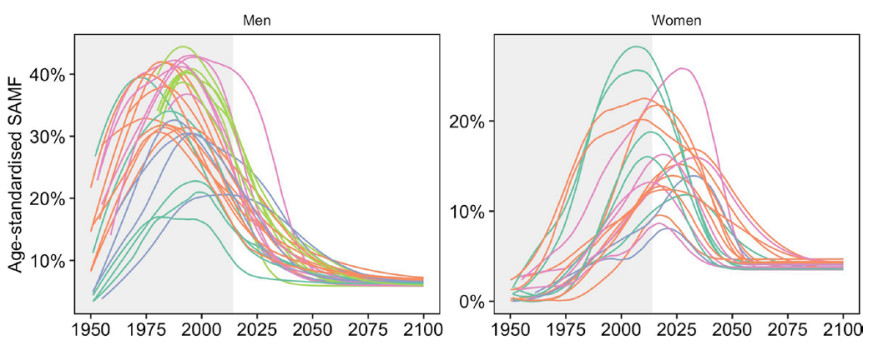

Figure 3 Past and future trends in smoothed* age-standardised smoking-attributable mortality fractions (SAMF) (men: 35-84; women: 40-84), 1950-2100, for the different countries compared, by sex. *Loess smoothing (degree $=2$; span $=0.25$ ) of fitted (or observed when absent) with projected.

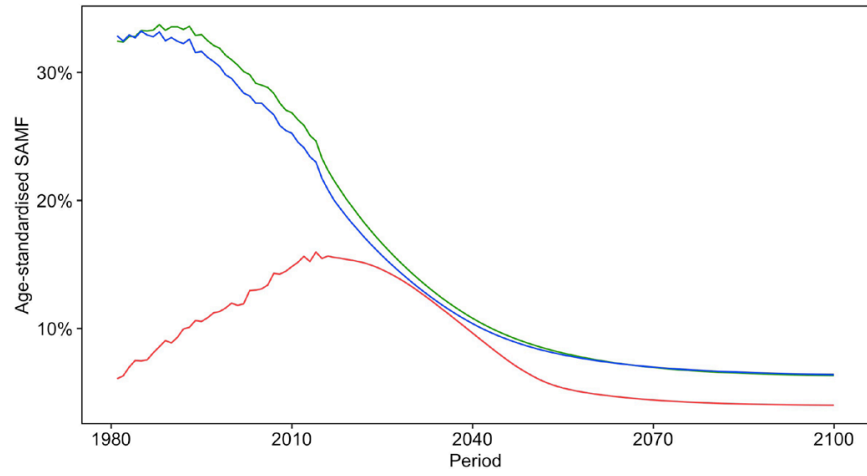

Figure 4 Unweighted average observed and projected agestandardised smoking-attributable mortality fractions (SAMF) (men: 35-84; women: 40-84) across 21/29 European countries* from 1981 to 2100. *For 1981-1984 Slovenia is not included in the average because of data unavailability. This did hardly affect the average. Because no reliable projections for women could be generated for Portugal, Spain and the six Eastern European countries, we also included the trends across the remaining 21 countries for men.

when projecting) to ensure that the drift (=shared linear trend between period and cohort) is completely removed from the cohort dimension, and added to the period dimension. Our method can also be applied using other APC models, ${ }^{38}$ although models with multiple period or cohort parameters will likely generate less transparent projections.

Failing to account for cohort effects when predicting smokingattributable mortality, as in the case of Li and Raftery, ${ }^{20}$ does not generate a realistic future age pattern for women, which is most likely why they did not present age-specific outcomes. Moreover, given the recent decline in the cohort parameter observed for women in all studied European countries and for men in the majority of countries, not accounting for cohort effects could lead to an overestimation of future levels and later peak years. Indeed, the peak years for women Li and Raftery projected were later than those in our projections for the majority of countries (online supplementary appendix table 2).

We implemented UBs and LBs in our methodology to obtain feasible values of the projected fractions (and PIs). More specifically, we avoided higher maximum SAMF levels for women than for men in each country, in line with the theory. ${ }^{3}$ In addition, we assumed a future minimum smoking prevalence of $5 \%$ for both men and women, based on a careful consideration of the available evidence. Although this level reflects an important further reduction in smoking prevalence from the current average level of $18 \%$ in Europe, ${ }^{28}$ reaching it seems feasible given the current smoking prevalence of $8.7 \%$ in Sweden, ${ }^{28}$ and the strong emphasis on smoking prevention policies in recent years. ${ }^{30-34}$ Given that women currently have higher smoking prevalence levels than men in selected forerunner countries like Sweden, ${ }^{28}$ we consider it unlikely that women will have lower minimum smoking prevalence levels than men. The finding that the percentage of heavy smokers is slightly higher among men than among women in Sweden, ${ }^{28}$ lines up nicely with the translation of the minimum smoking prevalence of $5 \%$ to slightly lower agestandardised SAMF minimum values for women (3.5\%) than for men $(5.9 \%)$.

Our sensitivity analysis (online supplementary file 1, page 18-23) revealed that while the implementation of the assumed UBs did not alter the point estimates, it diminished the width 
of the PIs for women. The implementation of the assumed LBs resulted-logically-in higher future SAMF, values in the long run compared with not implementing a LB (or, in other words, a LB that is just above zero) (online supplementary table S5). Importantly however, only minimal differences in the peak years and levels of $\mathrm{SAMF}_{t}$ for women were found, and with no uniform direction (online supplementary table S5 and figure S7). The future age patterns with and without the LBs (online supplementary figure S8) proved very similar for men, except in Ireland, Italy, Norway and particularly Sweden, where without the LBs, SAMF peaked at younger ages, reflecting very recent trends. For women, the peaks in the projected age patterns without the LBs occur, in the long run, at higher ages, even at higher ages than those for men. This finding likely reflects an unrealistic unbounded continued future decline of the cohort parameter.

Our projection outcomes-predominantly those in the very long run-are, thus, dependent on the selected LBs and their assumed age patterns, both of which rely on ongoing policy efforts to reduce smoking and its negative health consequences.

Our data-driven age-period-cohort approach, which projected the logit of the age-specific SAMF by means of a quadratic curve and implemented LBs, generated a very smooth asymmetric wave pattern, for example, for men in Portugal and women in Ireland, Luxembourg and Slovenia. But for women in the majority of countries, the wave changed rather abruptly from quadratic to level. This could imply an underestimation of the projected SAMF levels in the decades following the peak, in line with our validation results and those of $\mathrm{Li}$ and Raftery. ${ }^{20}$ This finding illustrates the difficulties associated with predicting the decelerating decline after the peak using a purely data-driven approach that does not include additional information and/or assumptions. This reflects the trade-off, in forecasting, between the objectivity and wide applicability of a purely data-driven approach and the advantages of adding subjective information, when available, to generate outcomes more in line with the expectations. ${ }^{39} 40$ Indeed, Stoeldraijer et al managed to obtain a smooth asymmetric pattern in line with the theory for Denmark, the Netherlands and the UK by employing a more qualitative APC approach. ${ }^{19}$ Their approach resulted in much later peaks for women in Denmark and the Netherlands (online supplementary appendix table 2) and higher values in 2050 than our current estimates. Our estimate for the Netherlands is much closer to that of Li and Raftery, ${ }^{20}$ who also employed an exclusively datadriven approach (no results for Denmark).

A few of our outcomes are not completely in line with the general expectations of the smoking epidemic model. ${ }^{3}{ }^{4}$ For women in the Czech Republic, Poland and Slovakia, the peak years are earlier than would be expected for Central European countries; and for women in Italy and Greece, the peak years are rather dissimilar. The results of $\mathrm{Li}$ and Rafter ${ }^{20}$ - who explicitly incorporated shared patterns between countries and between sexes-are more in line with the general theory for these countries (see online supplementary appendix table 2). This illustrates another important choice in forecasting: namely, of mainly relying on country-specific data, as we did; or of using, as Li and Raftery did, a multi-population approach in which the parameters are influenced by expected commonalities between countries and sexes. An important advantage of using a multi-population approach is a diminished reliance on the start year of the projections, ${ }^{18}$ which we found to be important for Greece. But given the important exceptions to the smoking epidemic model, ${ }^{5}$ using an approach that relies heavily on generalities might be tricky. Indeed, for women in the above-mentioned Central European countries, the data for (at least) the younger age groups clearly show evidence of stagnation (see online supplementary PDFs), indicating that our projected peak might be more realistic.

All in all, we believe our method represents a very promising approach for forecasting age-specific and age-standardised SAMF in the long run, and for projecting the peak in SAMF among women. Our projected levels can be used as a baseline to assess the influence of future specific public health actions. Our approach could also be used to forecast smoking-attributable mortality rates, and to forecast mortality from other factors that evolve as an epidemic (such as obesity), and in which the cohort dimension plays an important role.

\section{Overall conclusion}

Our novel forecasting method enabled us to forecast (agespecific) smoking-attributable mortality for the long-term future, and to obtain realistic estimates of the mortality imprint of the smoking epidemic in Europe up to 2100. While making optimal use of both the data and the theory, our projections are based on

\section{What this paper adds}

What is already known on this subject

- Past trends in smoking-attributable mortality show a clear (indication of) a wave pattern (increase followed by decline) that occurred earlier among men than women, and earlier in north-Western European countries than in other European countries, in line with the smoking epidemic model.

- Important differences between birth cohorts in smokingattributable mortality levels exist.

What important gaps in knowledge exist on this topic

- The few previous projections of smoking-attributable mortality into the long-term future for European countries were either based on semi-quantitative approaches that cannot be easily applied to many countries, or did not incorporate the cohort dimension, and therefore could not obtain realistic (age-specific) estimates.

\section{What this paper adds}

- The empirical extension of the smoking epidemic model up to recently, and realistic estimates of the mortality imprint of the smoking epidemic in Europe in the long-term future, by projecting (1) age-specific and age-standardised smokingattributable mortality fractions (SAMF), and their projection intervals, up to 2100 in 29 European countries, by sex and (2) the expected timing and level of the maximum mortality impact of smoking for women.

- For men in the 29 European countries studied, we project the continued deceleration of the decline in SAMF from the average peak of 35\% in 1989 and an average level of $25 \%$ in 2014 to $11 \%$ in $2040,7 \%$ in 2065 and $6 \%$ in 2100 . For women in the 21 non-Eastern European countries, with current average SAMF levels of $16 \%$, we project that the average peak in SAMF will be reached at a level close to half the level for men, and 30-35 years after the peak for men, after which SAMF will decline at a decelerating pace to, on average, $4 \%$ in 2100 .

- A novel approach to forecasting smoking-attributable mortality over the long term that is data-driven, and that integrates both the wave pattern of the smoking epidemic and the importance of the cohort dimension in the underlying model. 
the assumption that the current effective public health actions aimed at reducing (the negative effects of) smoking in Europe will, at least, continue.

The high projected peak values in smoking-attributable mortality for women in many European countries, and the finding that smoking-attributable mortality among women has already or will soon become higher than smoking-attributable mortality among men in selected European countries, warrant special attention from health policy-makers and society. In addition, the important country-differences in projected smokingattributable mortality-particularly among women—require attention.

Contributors FJ conceived the study. FJ and $A B$ designed the study. AB developed the projection model and performed the initial projections, with input from FJ. SEIG finalised the projections, with input from $A B$ and FJ. All authors interpreted the results. FJ drafted the manuscript. FJ and SEIG drafted the supplementary data and methods file. AB provided critical comments for both documents. FJ revised the documents. All authors approved the final versions of the documents.

Funding This work is funded by the Netherlands Organisation for Scientific Research (NWO) under grant no. 452-13-001. The funding source had no role in the study design, collection, analysis or interpretation of the data; in writing the manuscript or in the decision to submit the paper for publication.

Competing interests All authors have completed the ICMJE uniform disclosure form at http://www.icmje.org/coi_disclosure.pdf and declare: a grant from the Netherlands Organisation for Scientific Research (NWO) during the conduct of study; no other relationships or activities that could appear to have influenced the submitted work. AB is currently affiliated with the Nationale Nederlanden, but his work for the manuscript was as good as completed before he joined Nationale Nederlanden. He, subsequently, provided occasional expert advice on the methodology, and participated in the final critical read of the paper.

Patient consent for publication Not required.

Provenance and peer review Not commissioned; externally peer reviewed.

Data availability statement Data on lung-cancer mortality and on all-cause mortality are available online via the WHO Mortality Database and the Human Mortality Database, respectively. The data that was used as input for the tables and figures will be made available at Open Science Framework: https://osf.io/xspk8/. The $R$ codes can be requested from the author.

Open access This is an open access article distributed in accordance with the Creative Commons Attribution Non Commercial (CC BY-NC 4.0) license, which permits others to distribute, remix, adapt, build upon this work non-commercially, and license their derivative works on different terms, provided the original work is properly cited, appropriate credit is given, any changes made indicated, and the use is non-commercial. See: http://creativecommons.org/licenses/by-nc/4.0/.

ORCID iD

Fanny Janssen http://orcid.org/0000-0002-3110-238X

\section{REFERENCES}

1 WHO. Health in the European Union - trends and analysis. Copenhagen: World Health Organization - European Observatory on Health Systems and Policies, 2009

2 WHO Regional Office for Europe. Tobacco: data and statistics. Available: http:// www.euro.who.int/en/health-topics/disease-prevention/tobacco/data-and-statistics [Accessed 14 Mar 2019].

3 Lopez AD, Collishaw NE, Piha T. A descriptive model of the cigarette epidemic in developed countries. Tob Control 1994;3:242-7.

4 Thun M, Peto R, Boreham J, et al. Stages of the cigarette epidemic on entering its second century. Tob Control 2012;21:96-101.

5 Janssen F. Similarities and differences between sexes and countries in the mortality imprint of the smoking epidemic in 34 low-mortality countries, 1950-2014. Nicotine Tob Res 2020;22:1210-20

6 Janssen F, Kunst AE, Netherlands Epidemiology and Demography Compression of Morbidity research group. Cohort patterns in mortality trends among the elderly in seven European countries, 1950-99. Int J Epidemiol 2005;34:1149-59.

7 Preston SH, Wang H. Sex mortality differences in the United States: the role of cohort smoking patterns. Demography 2006;43:631-46.

8 Murray CJ, Lopez AD. Alternative projections of mortality and disability by cause 19902020: Global Burden of Disease Study. Lancet 1997;349:1498-504.

9 Mathers CD, Loncar D. Projections of global mortality and burden of disease from 2002 to 2030. PLoS Med 2006:3:e442.
10 Hughes $B B$, Kuhn R, Peterson CM, et al. Projections of global health outcomes from 2005 to 2060 using the International Futures integrated forecasting model. Bull World Health Organ 2011;89:478-86.

11 WHO. Projections of mortality and causes of death, 2012 to 2030, 2013. Available: http://www.who.int/healthinfo/global_burden_disease/projections/en/

12 WHO. Projections of mortality and causes of death, 2017 to 2060, 2018. Available: http://www.who.int/healthinfo/global_burden_disease/projections/en/

13 Foreman KJ, Marquez N, Dolgert A, et al. Forecasting life expectancy, years of life lost, and all-cause and cause-specific mortality for 250 causes of death: reference and alternative scenarios for 2016-40 for 195 countries and territories. Lancet 2018:392:2052-90.

14 Brennan P, Bray I. Recent trends and future directions for lung cancer mortality in Europe. Br J Cancer 2002:87:43-8.

15 Clements MS, Armstrong BK, Moolgavkar SH. Lung cancer rate predictions using generalized additive models. Biostatistics 2005:6:576-89.

16 Shibuya K, Inoue M, Lopez AD. Statistical modeling and projections of lung cancer mortality in 4 industrialized countries. Int I Cancer 2005;117:476-85.

17 Riebler A, Held L. Projecting the future burden of cancer: Bayesian age-period-cohort analysis with integrated nested Laplace approximations. Biom J 2017;59:531-49.

18 Janssen F, van Wissen LJG, Kunst AE. Including the smoking epidemic in internationally coherent mortality projections. Demography 2013;50:1341-62.

19 Stoeldraijer L, Bonneux L, van Duin C, et al. The future of smoking-attributable mortality: the case of England \& Wales, Denmark and the Netherlands. Addiction 2015:110:336-45.

20 Li Y, Raftery AE. Estimating and forecasting the smoking-attributable mortality fraction for both genders jointly in over 60 countries. Ann App/ Stat 2020;14:381-408.

21 Peto R, Lopez AD, Boreham J, et al. Mortality from tobacco in developed countries: indirect estimation from national vital statistics. Lancet 1992:339:1268-78.

22 Thun MJ, Day-Lally C, Myers DG, et al. Trends in tobacco smoking and mortality from cigarette use in Cancer Prevention Studies I (1959 through 1965) and II (1982 through 1988). In: Burns DM, Garfinkel L, Samet JM, et al, eds. Changes in cigaretterelated disease risks and their implications for prevention and control. Bethesda, Md: National Cancer Institute: Smoking and Tobacco Control Monograph, 1997: 305-82.

23 WHO. WHO Mortality Database. Health statistics and health information systems. Available: www.who.int/healthinfo/statistics/mortality_rawdata/ [Accessed 11 Apr 2018].

24 Human Mortality Database. University of California, Berkeley (USA), and Max Planck Institute for Demographic Research (Germany). Available: www.mortality.org [Accessed 29 Sep 2018].

25 Human Mortality Database. University of California, Berkeley (USA), and Max Planck Institute for Demographic Research (Germany). Available: www. mortality.org [Accessed 1 May 2019].

26 Cairns AJG, Blake D, Dowd K, et al. A quantitative comparison of stochastic mortality models using data from England and Wales and the United States. N Am Actuar J 2009;13:1-35

27 Juel K. Increased mortality among Danish women: population based register study. BMJ 2000;321:349-50

28 Eurostat. Tobacco consumption statistics. Available: https://ec.europa.eu/eurostat/ statistics-explained/index.php/Tobacco_consumption_statistics\#level_of_cigarette_ consumption [Accessed 14 Mar 2019].

29 IHME. Tobacco visualization. Available: http://vizhub.healthdata.org/tobacco/ [Accessed 14 Mar 2019].

30 WHO. Global action plan for the prevention and control of noncommunicable diseases 2013-2020. Geneva: World Health Organization, 2013.

31 Department of Health. Towards a Smokefree generation: a tobacco control plan for England. London: Department of Health and Social Care, 2017.

32 Dutch Government. National prevention agreement. The Hague: Ministry of Health, Welfare and Sports, 2018

33 Kavanagh P, Sheridan A. The state of tobacco control in Ireland. Dublin: HSE Tobacco Free Ireland Programme, 2018.

34 Feliu A, Filippidis FT, Joossens L, et al. Impact of tobacco control policies on smoking prevalence and quit ratios in 27 European Union countries from 2006 to 2014. Tob Control 2019;28:101-9.

35 Box GE, Jenkins GM. Time-series analysis, forecasting and control. San Fransisco: Holden-Day, 1976.

36 Reitsma MB, Fullman N, Ng M, et al. Smoking prevalence and attributable disease burden in 195 countries and territories, 1990-2015: a systematic analysis from the Global Burden of Disease Study 2015. Lancet 2017:389:1885-906.

37 Clayton D, Schifflers E. Models for temporal variation in cancer rates. II: age-periodcohort models. Stat Med 1987:6:469-81.

38 Yang Y, Fu WJ, Land KC. 2. A methodological comparison of age-period-cohort models: the intrinsic estimator and conventional generalized linear models. Sociol Methodol 2004;34:75-110.

39 Janssen F. Advances in mortality forecasting: introduction. Genus 2018;74:1-12.

40 Stoeldraijer L. Mortality forecasting in the context of non-linear past mortality trends: an evaluation. PHD thesis. ISBN: 978-94-034-1236-8. The Netherlands: University of Groningen, 2019. 